\section{Effects of familiarization with incongruous stimuli on their dominance}

in visual selection

TERRY T. FAW, University of California, Los Angeles, Calif. 90024, and JUM C. NUNNALLY, Vanderbilt University, Nashville, Tenn. 37203

The visual fixations of college students were recorded as they viewed eight pairs of stimulus drawings in which one element of each pair depicted an incongruous figure while the other element depicted a banal figure. For half of the Ss, the incongruous drawing in each pair was a simple variation of its banal counterpart, while for the other half, the incongruous drawings were unrelated to their banal counterparts. Prior to presenting the paired stimuli, $S$ was familiarized with the incongruous drawings in four of the eight stimulus pairs. The results indicate that: (1) the dominance in visual selection of incongruous stimuli does not result from Ss simply comparing the incongruous elements of the stimuli in a visual display, (2) familiarization with an incongruous drawing diminishes its potential to dominate visual selection, and (3) familiarization effect is greater when an incongruous stimulus is not a simple variation of its banal counterpart.

In an earlier study, Nunnally, Faw, \& Bashford (1969) reported an increasing monotonic relationship between stimulus incongruity and viewing time towards a stimulus. They further reported that the influence of incongruity on visual selection waned after 10 to $20 \mathrm{sec}$, and was effectively nonexistent at the end of a 30-sec exposure period. It was contended that the influence of incongruity on visual selection derived from the S's difficulty in resolving informational conflict in the incongruous stimuli. That is, a more incongruous figure was looked at longer than its less incongruous counterpart because it elicited more conflicting coding responses; thus, a longer time was required for $S$ to resolve the informational conflict. The decreased influence of incongruity on visual selection resulting from continued exposure to the visual display was attributed to the resolution of conflicting information in the stimuli, and the consequent equating of informational conflict among them.

In spite of the convincing evidence of an increasing relationship between stimulus incongruity and looking time, results from the aforementioned study were not entirely in keeping with the hypothesized process underlying visual selection. Based on the same reasoning as that which predicted the waning influence of incongruity with continued exposure to a stimulus display, one would expect that $S s$ who had previously been required to describe the experimental stimuli would subsequently exhibit little dominance of the incongruous stimuli over their banal counterparts. In the Nunnally et al study, such was not the case. The $S s$ who described the experimental stimuli prior to the experiment exhibited the same dominance of incongruous stimuli as was exhibited by Ss not previously given the opportunity to resolve informational conflicts inherent in the stimuli. In order to determine whether that unexpected result was attributable to specific characteristics of that previous study, and consequently unrepresentative of the general phenomenon, or was a replicable phenomenon not in accord with the proposed model of visual selection, the present experiment was designed to examine further the dominance in visual selection of preexposed incongruous stimuli.

In several previous studies, Ss have been shown pairs of stimuli in which the elements of the pair differed in their level of incongruity (e.g., Berlyne, 1958; Faw \& Nunnally, 1967). In each study, however, the incongruous stimuli compared were simple variations of the same banal counterpart (e.g., a cow, a cow with dots, a long-legged cow, and a cow with an elephant's trunk, might form one set of stimulus materials). Comparing stimuli having the same banal counterpart leaves open the possibility that Ss are simply comparing the incongruous elements of the two stimuli presented to them, rather than resolving informational conflict independently within each stimulus. A second purpose of the present experiment was to determine if that possibility was viable.

\section{SUBJECTS}

The Ss were 36 male and 36 female college students.

\section{APPARATUS}

The apparatus for recording eye movements is described in detail elsewhere (Faw \& Nunnally, 1967). It consisted of a viewing box, with a translucent screen at one end and a goggle-like opening at the other end. The $\mathrm{S}$ rested his head in the opening and viewed stimulus slides projected onto the screen at the far end of the box. A motion picture camera, mounted on the side of the box, photographed the movements of the S's left eye. A chin rest was used to reduce head movements, and $S$ wore ear protectors to reduce potentially distracting sounds.

\section{STIMULI}

The stimuli consisted of pairs of drawings photographed on $35-\mathrm{mm}$ slides and projected onto the viewing screen. The drawings were separated horizontally on the screen by at least $10 \mathrm{in}$. This separation forced $S$ to make a gross eye movement in order to bring either stimulus into his direct line of sight.

Eight pairs of line drawings were photographed as stimulus slides. Each pair consisted of a banal figure (e.g., a horse) and a corresponding incongruous figure (e.g., a horse with a man's head). Because the incongruous figure in each of those pairs was a simple variation of its banal counterpart, the eight stimuli are referred to as within-figure comparisons. Also photographed as stimulus slides were the 56 possible pairs of banal drawings and incongruous drawings in which the incongnuous drawing was not a simple variation of its banal counterpart. Those 56 stimulus pairs are referred to as between-figure comparisons.

The eight within-figure comparison stimuli were shown to half of the Ss, and the remaining Ss were shown eight stimulus pairs drawn from the collection of between-figure comparison stimuli. The paired stimuli shown each $S$ in that later group were selected at random from the pool of 56 stimulus pairs, with the following restrictions: (1) no banal stimulus or incongruous stimulus could appear in more than one pair; and (2) across all Ss, each banal stimulus and each incongruous stimulus was seen equally often.

\section{PROCEDURE}

To reduce the possibility of Ss simply responding to demand characteristics of the experiment, it was explained to $S$ that the experiment concerned potential changes in pupil size associated with different patterns of eye movements. The S was told that differentt arrangements of pictures would be projected onto the screen in the front of the box and that those arrangements had, in the past, been shown to produce the kind of eye-movement patterns of interest in the study (e.g., either small eye movements restricted to a small area or gross eye movements covering large areas). It was emphasized that what $S$ looked at during 


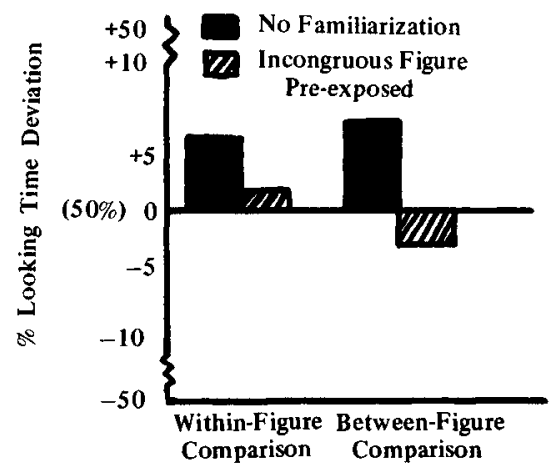

Fig. 1. Deviation of looking time percentages towards incongruous stimuli from expected value of $50 \%$.

the experiment was irrelevant to the study as long as he continued to look at the screen.

Following those instructions, $\mathrm{S}$ was shown a series of stimulus slides. The series consisted of eight paired drawings and four single drawings. Four of the eight paired drawings were randomly designated as those for which $\mathrm{S}$ would be familiarized with the incongruous element in the pair. For each of those four paired drawings a corresponding single drawing depicted its incongruous element. The presentation of that single drawing served to familiarize $S$ with the incongruous element in its corresponding pair.

For two of the four stimuli in each treatment condition, the incongruous stimulus was projected on the S's left, while for the other two pairs the incongruous stimulus was on his right. Both paired and single drawings were projected for $12 \mathrm{sec}$. The total presentation period was divided into two parts of $96 \mathrm{sec}$ duration each. During a period of approximately $45 \mathrm{sec}$ between parts, $S$ was allowed to take his head from the box and rest.

\section{ANALYSIS OF PHOTOGRAPHY}

Individual movie frames were analyzed by an experienced judge who was uninformed about the stimuli shown to $S$. The judge recorded the position of the eye fixation as either right, left, or uninterpretable (e.g., in the center or eye closed). Employing an identical procedure, an earlier study reported an interjudge agreement of $97.5 \%$ over a sample of 4,000 frames of film (Faw \& Nunnally, 1967).

The frequencies thus acquired were pooled over the four stimulus pairs within each of the two treatment conditions (e.g., having had or not had the incongruous element previously exposed). For each condition, the percentage of time spent viewing the incongruous drawings was computed. Uninterpretable fixations were not used in the computations.

\section{RESULTS}

The precentage scores for the two treatment conditions were employed in a 2 by 2 analysis of variance. The two factors of that analysis were (1) within- vs between-figure comparisons, (2) familiarization. That analysis revealed a significant main effect attributable to familiarization, $F(1,70)=32.85, \mathrm{p}<.001$. However, that main effect must be interpreted in light of a significant interaction of the familiarization factor and the within- vs between-figure comparison factor, $F(1,70)=4.69$, $p<.05$. Figure 1 depicts that interaction. For both types of comparison stimuli, analyses of the simple main effects indicate that familiarization with the incongruous element of a pair significantly reduced its potential to dominate looking behavior: within-figure, $F(1,70)=5.70, \quad p<.05$; between-figure, $F(1,70)=25.18, p<.01$.

For both within- and between-figure comparisons, incongruous drawings were viewed longer than banal drawings when $S$ was not familiarized with either drawing: within-figure, $t(35)=5.08, \quad p<.02$ between-figure, $\mathrm{t}(35)=6.35, \mathrm{p}<.01$. In contrast, an incongruous drawing previously exposed to $S$ was not viewed longer than its banal counterpart $(p>.05)$, and for between-figure comparisons it was viewed significantly less than the banal drawing, $t(35)=2.14, .01<p<.05$.

\section{DISCUSSION}

The results of the present experiment are in keeping with the hypotheses suggested by the earlier reports of Nunnally et al. With the Ss, procedures, and stimuli employed in the present study, it was clearly demonstrated that familiarization with an incongruous stimulus diminishes, and in some cases even reverses, the dominance in visual selection of that incongruous stimulus over a banal counterpart. The inability of Nunnally et al to obtain similar results might be attributable either to the Ss employed (children rather than adults), the method used to familiarize Ss with the stimuli, or some less obvious characteristic that was unique to that experiment.

In the present experiment, Ss were exposed to pairs of stimulus drawings in which either a banal drawing was paired with an incongruous drawing that was a simple variation of the banal stimulus (a man-horse with a man), or an incongruous drawing was paired with an unrelated banal drawing (a man-horse with a rabbit). With both classes of comparative stimuli nonfamiliarized incongruous stimul dominated their banal counterparts. The magnitude of that dominance was not significantly different for the two classes of stimuli. That result provides some support for the premise that Ss are not simply comparing the stimuli in a visual display with one another, but are, instead, examining the stimuli independently of one another.

While a familiarization effect was observed for both within- and between-figure comparison stimuli, the two classes of comparative stimuli did not produce identical patterns of visual selection. When $S$ was first familiarized with an incongruous drawing, and subsequently that drawing was paired with a banal drawing of which it was a simple variation, the two drawings were viewed for approximately the same amount of time. In contrast, when the familiarized incongruous drawing was compared with a banal drawing of which it was not a simple variation, the banal drawing was viewed longer.

It is interesting to speculate as to the basis of this observed difference in behavior. One might argue, for example, that familiarization with an incongruous drawing provides $S$ the opportunity to resolve informational conflict inherent in that incongruous drawing, and, at the same time, serves to familiarize him with the banal drawing of which it is a simple variation (its standard). As a result of this incidental familiarization, any informational conflict inherent in either the incongruous drawing or its standard banal counterpart will be resolved. Accordingly, the two drawings will subsequently be viewed equally as-long. This is the result observed with the within-figure comparisons of the present study. If, on the other hand, the familiarized incongruous drawing is compared to a banal drawing that is not its standard, whatever informational conflict is inherent in the banal drawing will result in its being looked at longer than the incongruous drawing for which the informational conflict has been resolved. This is the result observed with the between-figure comparisons of the present study.

This interpretation of the observed results from the present study rests on the assumption that informational conflict is inherent even in a banal drawing. That is, it takes some short time even to identify, or categorize banal drawings. The results of another experiment reported by the present authors (Faw \& Nunnally, 1969), as well as the traditional literature concerned with the familiarity effect in visual selection, lend support to that assumption. In those earlier experiments, banal drawings that had not been preexposed to $S$ were looked at longer than other banal drawings that had been preexposed. This result could be 
interpreted to suggest that some informational conflict, however small, was inherent in the banal stimuli, and that a familiarity effect was observed because the informational conflict inherent in one of the two banal stimuli was resolved during its preexposure.

While it is interesting to speculate as to the basis of the unpredicted result that was observed in the present experiment, and to search for evidence in support of various interpretations of that result, speculation is little more than an exercise in a posteriori theorizing. It will be an important role of future research to first verify the reliability of this initial observation, and then proceed to examine the adequacy of the various explanations that are proposed to explain it.

\section{REFERENCES}

BERLYNE, D. E. The influence of complexity and novelty in visual figures on orienting response. Journal of Experimental Psychology, $1958,55,289-296$.

FAW, T. T., \& NUNNALLY, J. C. The effects on eye movements of complexity, novelty and affective tone. Perception \& Psychophysics, $1967,2,263-267$.

FAW, T. T., \& NUNNALLY, J. C. The familiarity effect in visual selection: Satiation or information conflict resolution? Proceedings of the American Psychological Association, 1969.

NUNNALLY, J. C., FAW, T. T., \& BASHFORD, $M$. B. The effect of degrees of incongruity on visual fixations in children and adults. Joumal of Experimental Psychology, 1969, 81, 360-364.

\section{NOTE}

1. This study was supported, in part, by Research Grant HD-03083 from the National Institute of Child Health and Human Development to the second author and, in part, by NIMH Predoctoral Fellowship No. MH39863 to the first author. The authors wish to express their thanks to Mrs. Nancy Ator and Miss Evelyn Proctor for their assistance in this experiment.

\section{Social comparison, influence, and need achievement ${ }^{1}$}

\author{
PATRICIA L. SHAPIRO, 2 Pennsylvania \\ State University, University Park, $P$. \\ 16802
}

The experiment tested the hypotheses that (1) Ss whose motive to approach success (Ms) is greater than their motive to avoid failure (Mf) would join influenceable groups and (2) Ss whose Mf is greater than Ms would join groups providing social comparison. The Ss whose Ms $>$ Mf chose others whose opinions differed from their own to a greater extent than $S$ s whose Mf $>$ Ms. Although the data could be viewed as supporting the hypotheses, another interpretation discussed is that $S$ s for whom Ms $>M f$ accept a broader range of views for social comparison.

Testing one aspect of Festinger's (1954) social-comparison theory, Radloff (1961) has demonstrated that Ss who lack confidence in the correctness of their opinions will affiliate for opinion evaluation. Gordon (1966) has demonstrated that confident Ss will affiliate for the purpose of influencing others, and also that high- and low-confidence Ss chose to affiliate with different sorts of groups. The low-confidence $S$ prefers affiliation with groups composed of individuals who are likely to be correct and who agree with him. Festinger (1954) maintains that such groups provide social comparison and social reality, and should increase S's confidence in his correctness. The high-confidence $S$ prefers affiliation with others who know they are likely to be incorrect and who disagree with him. Gordon argues that such groups will be seen as good targets for influence since their low probability of correctness should result in low confidence and increased persuasability.

Two lines of evidence suggest that S's degree of motivation to approach success (Ms) and to avoid failure (Mf), as defined by Atkinson (1958), will affect S's decision to seek social comparison or to attempt influence. It is predicted that Ss whose Ms $>$ Mf will attempt influence for two reasons. First, Atkinson argues that $\mathrm{Ss}$ whose Ms $>$ Mf will seek out and perform at their best in achievement situations, situations in which good performance leads to success or in which performance will be evaluated against some standard of excellence. Situations in which influence is possible seem to provide achievement opportunities, particularly since successful influence provides evidence of successful leadership, which is definitely considered an achievement in American culture. Therefore, it is predicted that Ss whose Ms > Mf will affiliate with influenceable others. Secondly, Brody (1963) has demonstrated that Ss who are high in Ms are more confident of their correctness than are those low in Ms. It will be recalled that high-confident Ss seek to influence others. It is also predicted that those whose Mf $>$ Ms will seek social comparison and social reality. First, Atkinson argues that such Ss will avoid achievement situations, and second, as Brody demonstrated, their confidence is likely to be low. To increase their confidence, they should seek a group providing social comparison and social reality.

\section{PROCEDURE ${ }^{3}$}

In the first phase of the experiment, 62 male undergraduates at The Pennsylvania State University were given the Mandler Sarason Test Anxiety questionnaire (Mandler \& Sarason, 1952) to measure Mf and the Thematic Apperception Test (TAT) to measure Ms. The neutral instructions for the TAT (Atkinson, 1958) were read. Four pictures $(4,17 \mathrm{BM}, 13 \mathrm{~B}$, and 14) were each shown for $20 \mathrm{sec}$, and Ss were given $4 \mathrm{~min}$ to write each story. The E scored these for $\mathbf{n}$ Ach after establishing a reliability of .90 with the practice stories in Atkinson (1958). The Ss were divided into four groups, designated as follows: HL-high n Ach, low TAQ $(\mathrm{N}=15)$; HH-high n Ach, high TAQ $(\mathrm{N}=16)$; LL-low n Ach, low TAQ $(\mathrm{N}=16)$; LH-low n Ach, high TAQ $(\mathrm{N}=15)$. For each test, Ss scoring at or above the median ( 0 for the TAT, 190 for the TAQ) were assigned to the $H$ groups, and the others were assigned to the $\mathrm{L}$ groups. For HL Ss, Ms $>$ Mf, and for LH Ss, Mf $>$ Ms. Since the relative strengths of $\mathrm{Mf}$ and $\mathrm{Ms}$ for $\mathrm{HH}$ and LL Ss cannot be specified, these Ss were not further tested.

In the second phase of the experiment, $E$ used an intercom to communicate with Ss who were tested one, two, or three at a time in individual, sound-deadened cubicles. The Ss were told that they would make decisions on three case studies and 